\title{
Direct Observation of the Interpenetrated Spherulites by Atomic Force Microscopy
}

\author{
Yasuhiko TeradA, Takayuki IKEHARA, and Toshio NISHI \\ Department of Applied Physics, School of Engineering, \\ The University of Tokyo, 7-3-1 Hongo, Bunkyo-ku, Tokyo 113-8656, Japan
}

(Received June 6, 2000; Accepted July 5, 2000)

\begin{abstract}
Interpenetrated spherulites of poly(butylene succinate)/poly(vinylidene chloride-co-vinyl chloride) (PBSU/PVDCVC) blends were investigated by atomic force microscopy (AFM). AFM images showed that the lamellar population-density of PBSU was higher than that of PVDCVC in a blend. This enables the lamellae of PBSU to penetrate into PVDCVC spherulites. AFM images of the spherulitic border between PBSU and PVDCVC spherulites in a blend showed the change in the growth orientation of PBSU lamellae and the absence of the depression in the height profile. The results indicated that the PBSU lamellae penetrated along the PVDCVC lamellae. The lamellar populationdensity of PVDCVC spherulites penetrated by PBSU was higher than that of what were not penetrated by PBSU. KEY WORDS Interpenetrated Spherulites / Crystalline Polymer Blends / Atomic Force Microscopy / Poly(butylene succinate) / Poly(vinylidene chloride-co-vinyl chloride) /
\end{abstract}

Interpenetrated (or interlocked) spherulites ${ }^{1-3}$ are a superstructure which are occasionally formed in a few miscible pairs of crystalline polymers. Miscible poly(butylene succinate)/poly(vinylidene chloride-co-vinyl chloride) (PBSU/PVDCVC) blends ${ }^{2,3}$ show interpenetrated spherulites; the spherulites of PBSU do not stop growing when they collide with those of PVDCVC. An important factor to realize interpenetration is the simultaneous growth of the spherulites of the two components. Since they have a small difference in the melting point $T_{\mathrm{m}}$, both components simultaneously crystallize in an appropriate range of the crystallization temperature $T_{\mathrm{c}}$.

Only a few were examined for miscible pairs of crystalline polymers with different chemical structures. ${ }^{1-8} \mathrm{We}$ have been seeking those exhibiting simultaneous spherulitic growth. Recently, we reported the crystallization behavior and morphology of the spherulites of PBSU/ PVDCVC blends by differential scanning calorimetry and polarizing optical microscopy (DSC and OM). ${ }^{2,3} \mathrm{OM}$ studies revealed that the spherulites of neat PBSU showed a negative and bright birefringent pattern. The spherulites of PBSU in blends showed neither negative nor positive patterns that rotated by about $45^{\circ}$ from the positive and negative patterns. The spherulites of PVDCVC in blends showed a positive and dark pattern as neat spherulites of PVDCVC. In blends, the birefringent pattern of PVDCVC spherulites changed into that of PBSU after penetration of PBSU, and it was the evidence for the formation of interpenetrated spherulites. Based on the difference of the brightness in the birefringent pattern, we concluded that the interpenetration resulted from the difference in the lamellar populationdensity between PBSU and PVDCVC spherulites. However, the evidence of these findings on the lamellar scale was not obtained because of the limited resolution of OM. Non-contact atomic force microscopy (AFM), on the other hand, enables one to visualize submicrometerscale spherulitic structures down to the level of a lamella. ${ }^{9-13}$

The purpose of the present paper is to show the evidence of interpenetration by non-contact AFM. Further- more, we will discuss the difference in the lamellar density and investigate the morphology of the blended PBSU spherulite with neither negative nor positive birefringence.

\section{EXPERIMEMT}

PBSU (Showa Denko) with $M_{\mathrm{w}}=140000, T_{\mathrm{g}}=241 \mathrm{~K}$, and $T_{\mathrm{m}}=387 \mathrm{~K}$ is a biodegradable crystalline polymer. PVDCVC (Asahi Chemical) with $M_{\mathrm{w}}=100000, T_{\mathrm{g}}=$ $279 \mathrm{~K}$, and $T_{\mathrm{m}}=421 \mathrm{~K}$ is a crystalline random copolymer of vinylidene chloride containing $20 \%$ vinyl chloride as a comonomer. They were dissolved into a mutual solvent, $N, N$-dimethylformamide, at about $370 \mathrm{~K}$. The solution was cast onto a glass plate. After the films were dried in air, they were kept in a vacuum chamber for several days at room temperature (RT) in order to remove residual solvent.

Interpenetrated spherulites were formed under the next condition; the PBSU content of $40 \%$ and $T_{\mathrm{c}}=353 \mathrm{~K}$, where (i) PBSU and PVDCVC nucleate almost simultaneously, (ii) the spherulitic growth rate of PBSU is slightly ( two times) higher than that of PVDCVC, and (iii) the nucleation frequency of PBSU is lower than that of PVDCVC. ${ }^{3}$

The samples were isothermally crystallized. After melted at $443 \mathrm{~K}$ for $10 \mathrm{~min}$ with a temperature controller (Linkam LK-600PM), the samples were quenched to $353 \mathrm{~K}$ at a rate of $-100 \mathrm{~K} \mathrm{~min}^{-1}$. After crystallized for $100 \mathrm{~min}$ they were subsequently quenched to $254 \mathrm{~K}$ to stop crystallization and fix the morphology of spherulites.

The morphology of the spherulites was observed at RT under crossed nicols with a polarizing microscope (Olympus BHA-P). Since the small birefringence of PVDCVC makes optical microscopic observation difficult, a sensitive-tint retardation plate was placed in the beam at $45^{\circ}$ to the directions of the crossed polarizer and analyzer.

AFM images were acquired at RT in non-contact mode using JEOL JSPM-4200. The cantilevers 


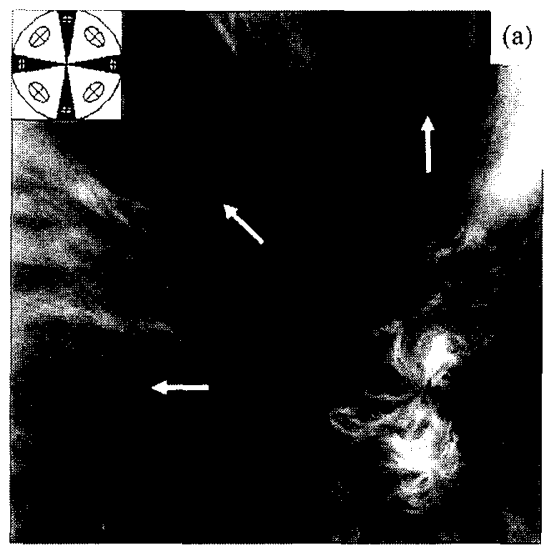

(a)

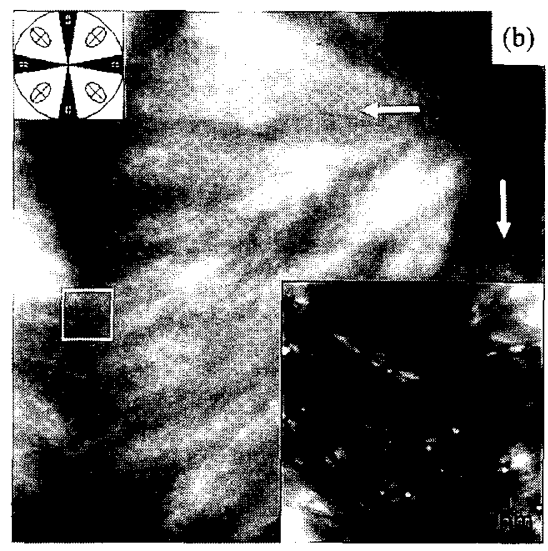

Figure 1. AFM images of neat (a) PBSU and (b) PVDCVC. Each birefringent pattern which is derived from the OM image is schematically shown in the top left inset. The lamellar orientations, which are determined by a magnified image, are indicated by the white arrows. In (b), the magnified image of the squared area is shown in the bottom right inset

(OMCL-AC120TS-1, Olympus) had a spring constant of $11--23 \mathrm{~N} \mathrm{~m}^{-1}$ and a resonance frequency of $248-330 \mathrm{kHz}$. The same area of a sample was observed by $\mathrm{OM}$ and $\mathrm{AFM}$.

\section{RESULTS AND DISCUSSION}

The AFM images of neat PBSU and PVDCVC spherulites crystallized at $363 \mathrm{~K}$ are shown in Figure 1. The topographic contrast of AFM images results from the lamellae or lamellar stacks (fibrils). ${ }^{9-13}$ In Figure 1a, the bright and dark regions were assigned to fibrils and uncrystallized (amorphous) regions, respectively. The fibrils of neat PBSU grew radially. In Figure $1 \mathrm{~b}$, the neat PVDCVC spherulite also consisted of radiating fibrils though they were unclear. The inset of (b) shows that there was a considerable amount of amorphous regions. This indicates that the crystallinity of PVDCVC is low, as indicated by low DSC melting peaks. ${ }^{2}$ This is reasonable because PVDCVC is a copolymer.

The OM observation of a blended sample is displayed in Figure 2a. It shows dark and bright spherulites. When the spherulites $\mathrm{A}, \mathrm{S} 1, \mathrm{~S} 2, \mathrm{~B} 1$, and B2 nucleated almost simultaneously, A was bright and the others were dark in the OM image. Since the birefringence of PBSU is larger than that of PVDCVC, A and the others are assigned to PBSU and PVDCVC spherulites, respectively. When the fast-growing A (PBSU) collided with the

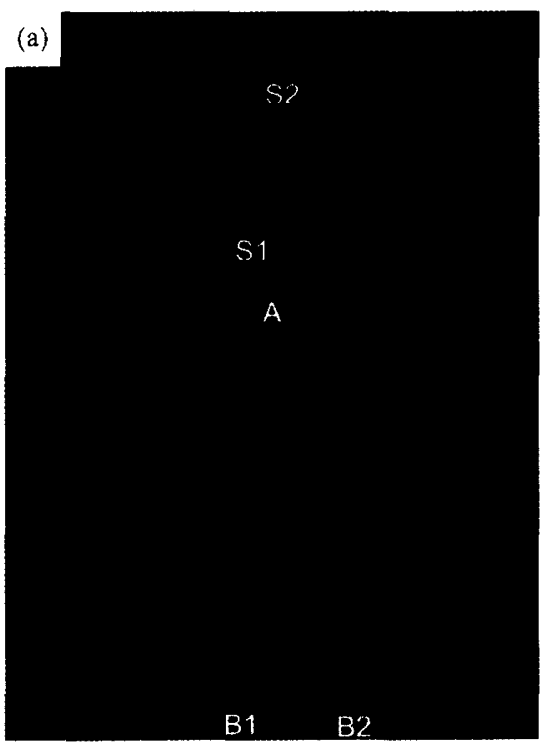

(b)
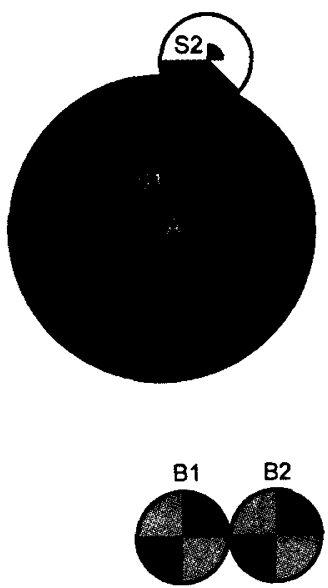

Figure 2. (a) OM image of a PBSU/PVDCVC $=40 / 60$ blend showing a PBSU spherulite (A) which penetrated through (S1) and into (S2) PVDCVC spherulites. $T_{\mathrm{c}}=353 \mathrm{~K}$ and crystallization time $=100 \mathrm{~min} .(\mathrm{b})$ is the schematic of $(\mathrm{a})$.

slowly-growing S1 (PVDCVC), the border of S1 became brighter. Then, the bright area spread toward the opposite border through the center of $\mathrm{S} 1$, as indicated by the partly penetrated spherulite S2 in Figure 2a. After A penetrated through $\mathrm{S} 1$, it continued to grow. The details of the penetration process were discussed in the previous study. $^{3}$

Figure $2 \mathrm{~b}$ shows the schematic birefringent patterns of Figure 2a. As in the previous work, ${ }^{3}$ the birefringent pattern of A was neither negative nor positive, and that of $\mathrm{S} 1$ was negative. On the other hand, that of $\mathrm{B} 1$ was unclear because of the small birefringence. This is possibly caused by the thin film thickness. The previous results, however, revealed that the birefringent pattern of PVDCVC spherulites in blends was positive as neat PVDCVC before penetration of PBSU. As shown in the previous work, penetration is confirmed by the change in birefringence of S1. Spherulites of neat PVDCVC showed a positive pattern, while the observed negative pattern of $\mathrm{S} 1$ resulted from the large birefringence of PBSU lamellae which grew along PVDCVC lamellae. 

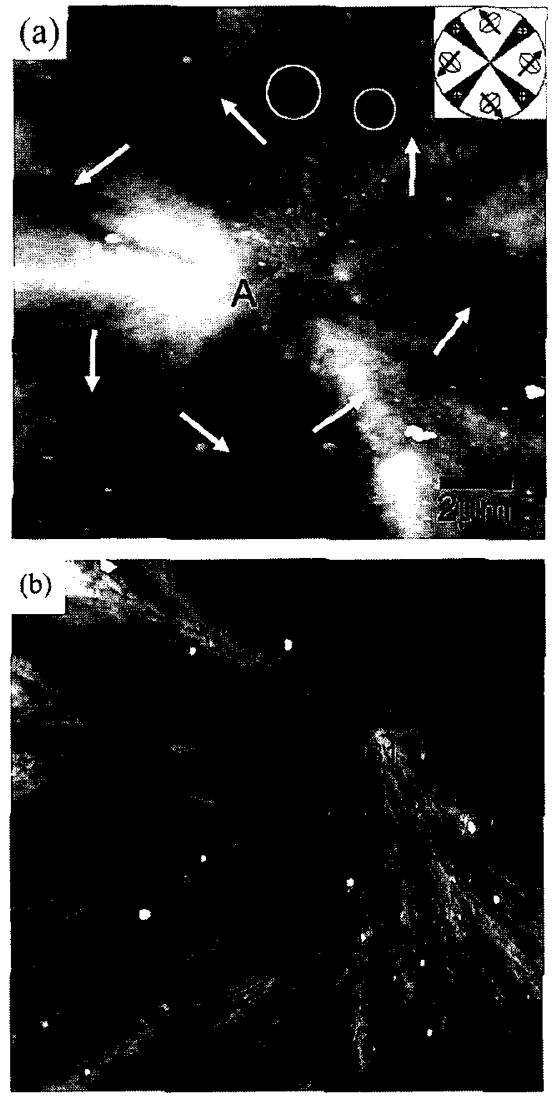

Figure 3. AFM images of the center of (a) A and (b) B1 spherulites in Figure 2. The birefringent pattern of a blended PBSU spherulite is schematically shown in the inset of (a). The lamellar orientations are indicated by the white arrows. The white circles in (a) point to the same positions as those in Figures $4 \mathrm{~b}$ and $4 \mathrm{c}$. The black arrows in the inset of (a) are drawn parallel to the short axes of the birefringent ellipsoids.

AFM images of the center of spherulites A (PBSU) and B1 (PVDCVC) are shown in Figures $3 \mathrm{a}$ and $3 \mathrm{~b}$, respectively. They are distinguished from each other by the orientation and population-density of lamellae. First, the lamellar orientation of PBSU was not parallel to the radial direction. It uniformly inclined by about $45^{\circ}$ from the radial direction as indicated by the arrows in Figure 3a. It explains the birefringence of the blended PBSU spherulite. As shown in Figure 1a, the lamellae of neat PBSU spherulites oriented parallel to the short axes of the birefringent ellipsoids. Therefore, the inclination of PBSU lamella in Figure 3 a results in the birefringent pattern which is neither negative nor positive shown in the inset. The inclination was not observed for neat PBSU spherulites. Although it is an interesting phenomenon, the origin of inclination is still under study. In contrast, the lamellar orientation of PVDCVC is almost parallel to the radial direction in Figure $3 \mathrm{~b}$ as the spherulites of neat PVDCVC.

Second, the lamellar density of A (Figure 3a) was higher than that of B1 (Figure 3b); the lamellae tightly filled the space. In contrast, B1 was sparser than A because of the low crystallinity of PVDCVC. This is the experimental evidence of the previously proposed model ${ }^{3}$ where the lamellar density of PVDCVC is lower than that of PBSU in blends.

The penetration of PBSU into PVDCVC results from 902
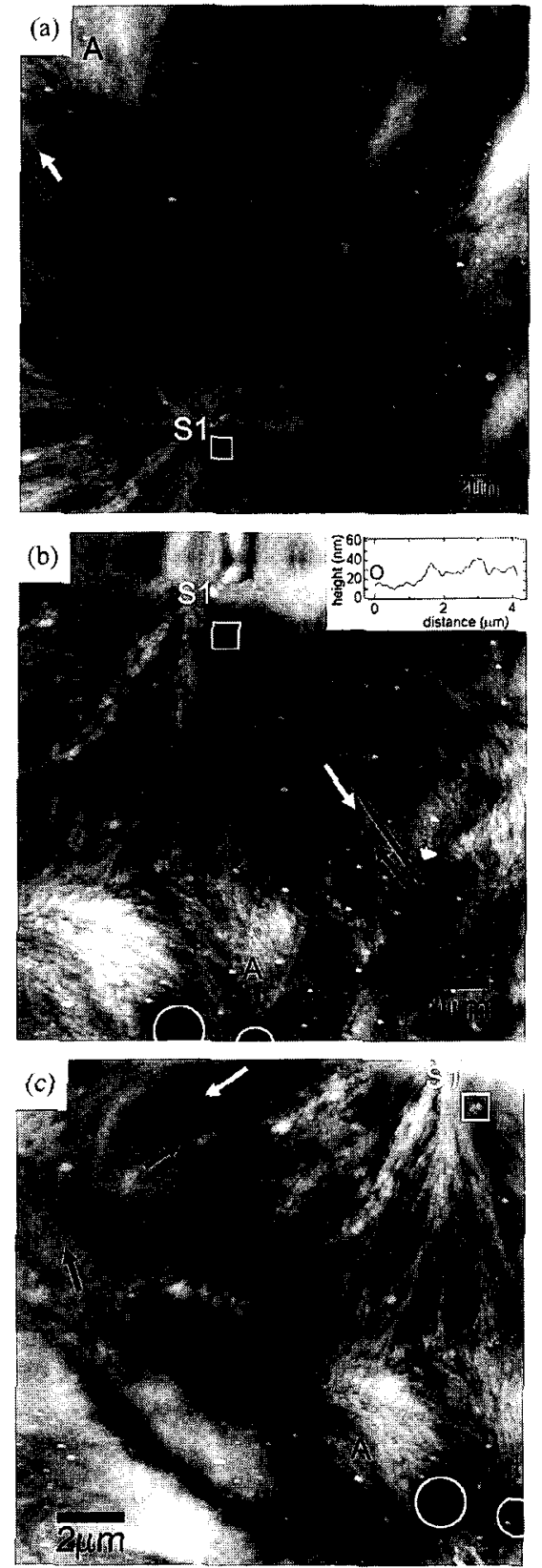

Figure 4. AFM images of S1 in its (a) upper, (b) lower right, and (c) lower left areas. The white squares in (a), (b), and (c) point to the same position near the center of S1. The white circles in (b) and (c) point to the same position as those in Figure 3a. The growth orientations of lamellae of PBSU and PVDCVC are indicated by the black and white arrows, respectively. In (b), the height profile along the black line is shown in the inset.

the low density of the lamellae in PVDCVC spherulites. As shown in Figure $3 \mathrm{~b}$, the lamellar density of a PVDCVC spherulite in a blend was sparse before penetration. This indicates that the PVDCVC spherulite contains a sufficient amount of amorphous PBSU in the interlamellar or interfibrillar regions before penetration. This enables the lamellae of PBSU to penetrate into PVDCVC spherulites. As shown by Figures $3 \mathrm{~b}$ and $4 \mathrm{a}$, the lamellar density of S1 (Figure 4a) was higher than that of B1 (Figure 3b). The lamellar densities of S1 and B1 must have been the same before penetration because they started to crystallize almost simultaneously. Thus, 


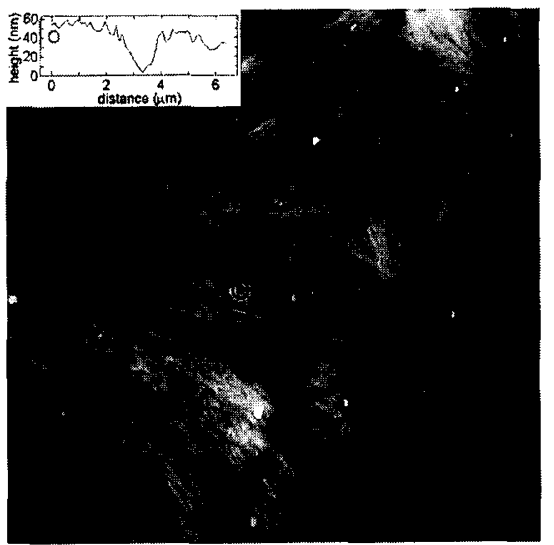

Figure 5. The depression in an AFM image of the border between PVDCVC spherulites (B1 and B2). The height profile along the black line is shown in the inset.

the higher lamellar density in S1 was caused by the penetration of A into S1.

The lamellar orientation at the border of $\mathrm{S} 1$ characterizes penetration (Figure 4). As shown in Figure 2, S1 is located just above the spherulite A. The lamellae of $\mathrm{A}$ grew upwards in Figures $4 \mathrm{~b}$ and $4 \mathrm{c}$. In the lower right area of S1 (Figure 4b), the lamellar orientations of A and $\mathrm{S} 1$ were almost the same. Thus, the lamellae of $\mathrm{A}$ smoothly penetrated along those of S1. In contrast, in the lower left area of S1 (Figure 4c), A and S1 lamellae oriented almost perpendicular to each other. In this case, A lamellae were observed to change the growth direction to penetrate into $\mathrm{S} 1$.

Figure $4 \mathrm{a}$ shows the regularly radiating lamellae in the upper area of S1. This indicates that A lamellae continued to grow along those of S1 after they passed the center of S1. The similar observation was obtained in the OM image of S2 (Figure 2) which showed the fan-shaped pattern of $\mathrm{A}$ in the upper right area of S2. In addition, Figure 4a shows the inclination of A lamellae in the upper area of the border of S1. This indicates that A lamellae inclined again after penetration as before penetration.

The height profile around the border between $\mathrm{S} 1$ and A also characterizes penetration. Figure $1 \mathrm{~b}$ showed the depression on the border between neighboring spherulites of neat PVDCVC. It was also present on the border between B1 and B2 (Figure 5). This is typical for crystalline polymer systems. ${ }^{11,13}$ The presence of the depression characterizes the termination of lamellar growth due to collision. However, the depression was not observed at the border between $\mathrm{S} 1$ and A (Figure $4 \mathrm{~b}$ ). This indicates that lamellae of A kept growing when they collided with S1.

There was no evidence for the penetration of PVDCVC into PBSU. The OM observation revealed that the growth front of a PVDCVC spherulite did not grow after collision with a PBSU spherulite. One possible explanation for this is the difference in crystallinity of the two specimens. Since AFM images of PBSU spherulites showed that they have few interlamellar or interfibrillar gaps observable by AFM before collision, the formation of the lamellae of PVDCVC in PBSU spherulites would hardly occur.

In summary, we investigated the morphology of interpenetrated spherulites in PBSU/PVDCVC blends by non-contact AFM. The AFM images of PBSU and PVDCVC spherulites in blends showed that PBSU was dense and PVDCVC was sparse. This causes the penetration of PBSU lamellae into PVDCVC spherulites. The lamellar population-density of a penetrated PVDCVC spherulite was higher than that of a non-penetrated one. Furthermore, the lamellar orientaion of PBSU was inclined by about $45^{\circ}$ from the radial direction, while that of PVDCVC was parallel to the radial direction. AFM images of the spherulitic border between PBSU and PVDCVC spherulites showed (i) the change in the lamellar orientation of PBSU and (ii) the absence of the depression in the height profile. There was no evidence for the penetration of PVDCVC lamellae into PBSU spherulites.

Acknowledgment. The authors would like to thank Showa Denko and Asahi Kasei for providing us of PBSU and PVDCVC, respectively. We are also grateful to Prof. K. Ito and Dr. T. Shimomura for their help to the present work.

\section{REFERENCES}

1. E. Blümm and A. J. Owen, Polymer, 36, 4077 (1995).

2. J.-C. Lee, H. Tazawa, T. Ikehara, and T. Nishi, Polym. J., 30, 780 (1998).

3. T. Ikehara and T. Nishi, Polym. J., 32, 683 (2000).

4. J.-C. Lee, H. Tazawa, T. Ikehara, and T. Nishi, Polym. J., 30, 327 (1998).

5. J.-C. Lee, H. Tazawa, T. Ikehara, and T. Nishi, J. Appl. Poly. Sci., B, Polym. Phys., 35, 2645 (1997).

6. J. P. Penning and R. St. John Manley, Macromolecules, 29, 77 (1996).

7. J. P. Penning and R. St. John Manley, Macromolecules, 29, 84 (1996).

8. K. Fujita and T. Kyu, Macromolecules, 29, 91 (1996).

9. J. K. Hobbs, T. J. McMaster, M. J. Miles, and P. J. Barham, Polymer, 39, 2437 (1998).

10. D. A. Ivanov, B. Nysten, and A. M. Jonas, Polymer, 40, 5899 (1999).

11. R. Pearce and G. J. Vancso, J. Polym. Sci., B, Polym. Phys., 36, 2643 (1998).

12. J. M. Schultz and M. J. Miles, J. Polym. Sci., B, Polym. Phys., 36, 2311 (1998).

13. H. R. Harron, R. G. Pritchard, B. C. Cope, and D. T. Goddard, J. Polym. Sci., B, Polym. Phys., 34, 173 (1996). 